\title{
A Rotavirus Virus-Like Particle Confined Palladium Nanoreactor and Its Immobilization on Graphene Oxide for Catalysis
}

\author{
Jie Zhu ${ }^{1,2} \cdot$ Xiaoxue $\mathrm{Lu}^{1} \cdot$ Yijian $\mathrm{Li}^{3} \cdot$ Tingdong $\mathrm{Li}^{3} \cdot$ Linsong Yang ${ }^{1} \cdot \mathrm{Kun} \mathrm{Yang}^{1} \cdot \mathrm{Liang} \mathrm{Ji}^{1} \cdot \mathrm{Mohong} \mathrm{Lu}^{2} \cdot \mathrm{Mingshi}^{\mathrm{Li}}{ }^{2}$
}

Received: 29 January 2020 / Accepted: 3 May 2020 / Published online: 14 May 2020

(C) Springer Science+Business Media, LLC, part of Springer Nature 2020

\begin{abstract}
In this work, a new viral protein cage based nanoreactor was successfully constructed via encapsulating Tween 80 stabilized palladium nanoparticles (NPs) into rotavirus capsid VP2 virus-like particles (i.e. Pd@ VP2). The effects of stabilizers including CTAB, SDS, Tween 80 and PVP on controlling the particle size of Pd NPs were investigated. They were further immobilized on graphene oxide (i.e. Pd@VP2/GO) by a simple mixing method. Some characterizations including FT-IR and XPS were conducted to study adsorption mode of Pd@VP2 on GO sheets. Their catalytic performance was estimated in the reduction of 4-nitrophenol (4-NP). Results showed that Tween 80 stabilized Pd NPs with the molar ratio of Pd to Tween 80 at 1:0.1 possessed the smallest size and the best stability as well. They were encapsulated into viral protein cages (mean size $49 \pm 0.26 \mathrm{~nm}$ ) to assemble confined nanoreactors, most of which contained 1-2 Pd NPs (mean size 8.15 $\pm 0.26 \mathrm{~nm}$ ). As-prepared Pd@VP2 indicated an enhanced activity (apparent reaction rate constant $k_{a p p}=(3.74 \pm 0.10) \times 10^{-3} \mathrm{~s}^{-1}$ ) for the reduction of 4-NP in comparison to non-confined Pd-Tween80 colloid $\left(k_{a p p}=(2.20 \pm 0.06) \times 10^{-3} \mathrm{~s}^{-1}\right)$. It was logically due to confinement effects of Pd@VP2 including high dispersion of Pd NPs and high effective concentration of substrates in confined space.Pd@VP2 were further immobilized on GO surface through C-N bond. Pd@VP2/GO exhibited good reusability after recycling for four runs, confirming the strong anchoring effects of GO on Pd@VP2.
\end{abstract}

Electronic supplementary material The online version of this article (https://doi.org/10.1007/s10562-020-03252-6) contains supplementary material, which is available to authorized users.



1 National-Local Joint Engineering Research Center of Biomass Refining and High-Quality Utilization, Changzhou University, Changzhou 213164, China

2 Jiangsu Key Laboratory of Advanced Catalytic Materials and Technology, Changzhou University, Changzhou 213164, China

3 State Key Laboratory of Molecular Vaccinology and Molecular Diagnostics, National Institute of Diagnostics and Vaccine Development in Infectious Diseases, School of Public Health, Xiamen University, Xiamen 361102, China 


\section{Graphic Abstract}

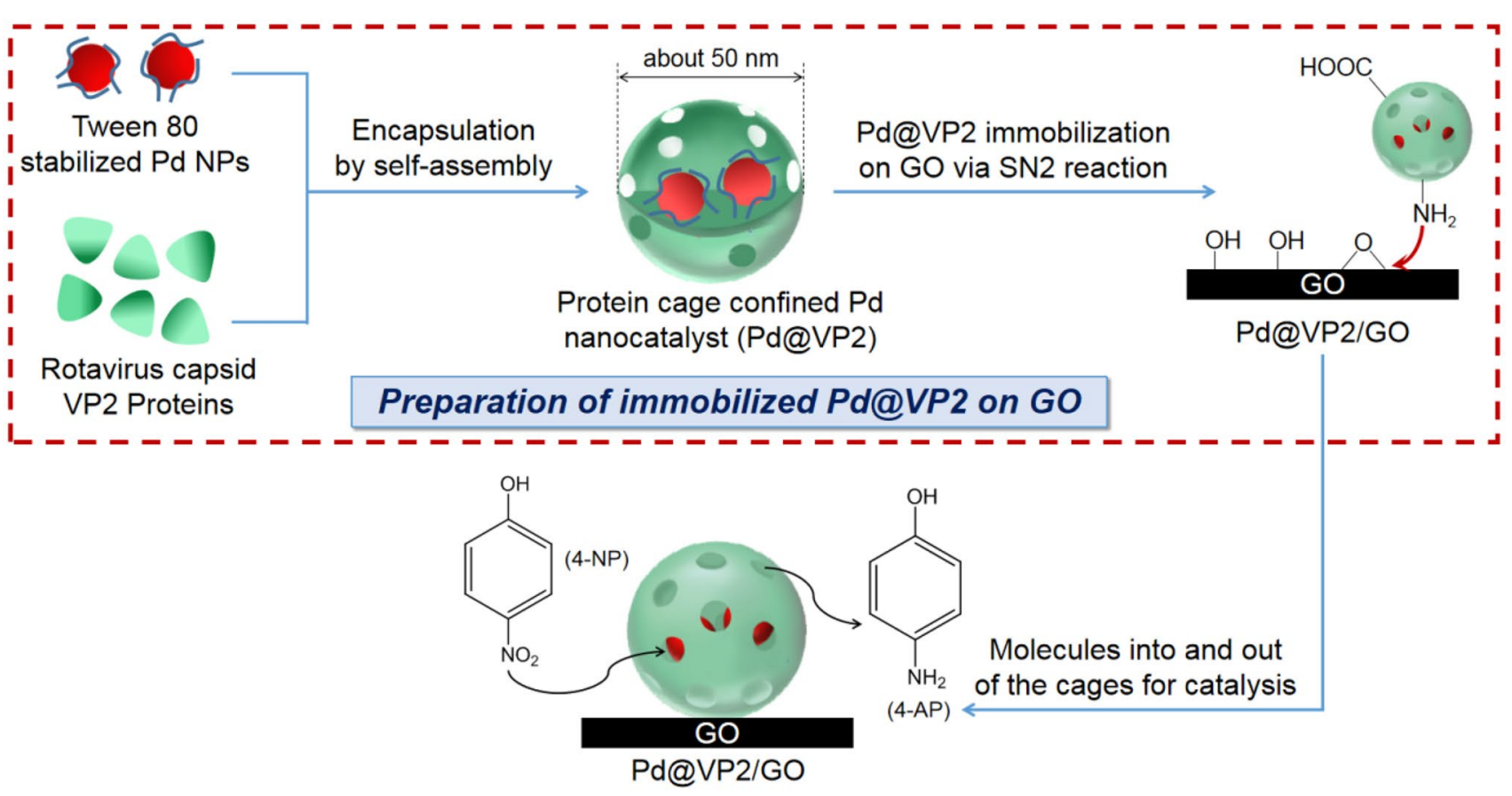

\section{Nitrophenol reduction over Pd@VP2/GO}

Keywords Rotavirus · Virus-like particles $\cdot$ Confined nanoreactor $\cdot$ Graphene oxide $\cdot$ Immobilization

\section{Introduction}

Metal catalysis within a nanoscopic confinement environment (e.g. nanocage and nanotube) has caught enormous attention very recently due to its superiority over non-confined analogues, such as maintaining high dispersion and stability of metal nanoparticles (NPs), imposing steric hindrance on catalyst-bound reactants and enhancing effective concentration of substrates in confined space [1-8]. These positive confinement effects remarkably improve the catalytic performance in the reaction.

To date, several chemically synthesized nanoscopic confinement systems, such as zeolites [9-11], carbon nano materials [12-15], polymers [16-18], metal organic frameworks (MOFs) [19-21] and micelles [22-24], have been developed as nanoreactors for various catalytic reactions. However, these confinement systems meet some challenges. Metal nanoparticles, for example, are hard to recycle from zeolites and MOFs due to the poor disassembled properties of those supports. Also, the size and morphology of micelles is difficult to effectively control in preparation process. Undoubtedly, these defects restricted their wide applications in sustainable chemistry and green chemistry.
Compared with chemically synthesized confinement systems, biological supramolecular ones, especially protein nanocages, have several significant characterization featuring reproducible, robust and monodisperse hollow scaffolds with highly organized structures [25]. Virus-like particles (VLPs), a class of protein cages, are of self-assembled hollow structures from viral coat proteins (CPs). As a new type of natural confinement structure, VLPs are often employed to encapsulate some external guest molecules (e.g. polymers, nanoparticles and proteins) to construct functionalized nanocages for drug delivery, biosensing and especially catalysis [26-30]. Liu et al. [30] encapsulated commercial Au NPs into cowpea chlorotic mottle virus (CCMV) protein cages with the sizes of $18 \mathrm{~nm}$. This confined nano catalyst exhibited a high catalytic activity in nitroarenes reduction.

Unfortunately, due to the highly dispersed nature of VLPs, the required separation of product and VLPs as well as VLPs recovery limited their applications. This issue could be addressed by immobilizing VLPs on solid supports such as silica [31], thereafter serving as heterogeneous catalysts for the reactions. At present, protein immobilization process often involves two steps including surface modifications (e.g. amine functionalization) on solid supports and further covalent combinations of proteins to them via amide, ester 
or ether linkages $[32,33]$. Obviously, this process is fussy and environment-unfriendly due to the use of toxic organic solvents and coupling agents.

Graphene oxide (GO), a promising candidate for VLPs immobilization, takes several advantages over other solid supports such as the well-dispersion nature in water, large surface area, small mass transfer limitations and low manufacturing cost $[34,35]$. More importantly, GO is endowed with abundant epoxy groups $(\mathrm{C}-\mathrm{O}-\mathrm{C})[36,37]$ that can directly react with amino groups $\left(-\mathrm{NH}_{2}\right)$ on VLPs under the mild conditions, resulting in the immobilization of VLPs on GO surface via a simple and green process without any coupling agents and organic solvents.

In our previous work [38], we constructed a genetically engineered strain Escherichia coli. (E. coli) BL21 (DE3) for the expression of rotavirus capsid VP2 proteins in the field of vaccine development. 120 copies of VP2 monomers can high-efficiently assemble to a spherical VLP with the outer size of $54 \mathrm{~nm}$ and the inner one of $45 \mathrm{~nm}$. This large protein cage helps the encapsulation of various cargoes at the same time in it. Noted that, VP2 VLP possesses 60 pores with a diameter of about 2-3 nm on its surface, conducive to the diffusion of guest molecules into and out of the cages for catalysis.

Herein, for the first time, we successfully encapsulated Pd nanoparticles in rotavirus VP2 VLPs by self-assembly. This confined nanocatalyst was further immobilized on GO for catalyzing the reduction of 4-nitrophenol (4-NP). Apparently, this study created a simple and environment-friendly route to develop immobilized VLPs confined catalysts for highly efficient aqueous reactions, which further broaden their application in sustainable and green chemistry.

\section{Experimental}

\subsection{Synthesis of Pd Nanoparticles}

Pd NPs were synthesized via colloidal method. Some ligands including cetyltrimethyl ammonium bromide (CTAB, Sinopharm, China), Sodium dodecyl sulfate (SDS, Sinopharm, China), Tween 80 (Sinopharm, China) and polyvinylpyrrolidone (PVP, average $\mathrm{MW}=10,000$, Sinopharm, China) are employed to maintain the stability of synthesized Pd NPs. In a typical experiment, Tween $80(0.1 \mu \mathrm{mol})$, for example, was firstly dissolved in water $(3 \mathrm{ml})$ at room temperature. Aqueous solution of $\mathrm{Na}_{2} \mathrm{PdCl}_{4}(78 \mu \mathrm{L}$, containing $2.0 \mu \mathrm{mol} \mathrm{Pd}$ ) was then mixed in above freshly prepared Tween 80 solution with stirring for $15 \mathrm{~min}$ under the protection of argon. After that, $\mathrm{NaBH}_{4}$ solution $(200 \mu \mathrm{L}, 30 \mu \mathrm{mol})$ was further added in it to reduce $\mathrm{Pd}^{2+}$ for 5 min under stirring. Finally, $\mathrm{Pd}$ colloid solution was formed. The preparation procedures of Pd colloids with other ligands including CTAB, SDS and PVP were similar to that of Tween 80 . In the procedure, the molar ratio of Pd to ligand was kept fixed at 20:1 (mol:mol). The size distribution of Pd colloidal NPs was measured here to investigate the effects of ligands on maintaining the stability of synthesized Pd NPs. The best ligand among four ones was selected to further optimize $\mathrm{Pd}$ colloid preparation via varying the molar ratio of $\mathrm{Pd}$ to this ligand.

\subsection{Encapsulation of Pd NPs into Rotavirus VP2 VLPs}

Recombinant rotavirus capsid VP2 proteins were expressed and purified according to a method described in our previous literature [38]. Ligands modified Pd NPs were encapsulated into rotavirus VP2 VLPs by assembling VP2 capsid proteins around Pd NPs. In a typical experiment, $100 \mu \mathrm{L}$ of Pd colloid solution (about $0.035 \mathrm{mg} / \mathrm{mL}$ in water) was injected to $1.0 \mathrm{~mL}$ of pre-purified rotavirus VP2 capsid protein solution $(0.25 \mathrm{mg} / \mathrm{mL})$ in Tris-HCl buffer $(50 \mathrm{mM}$ Tris-HCl, $\mathrm{pH} 8.0$, $500 \mathrm{mM} \mathrm{NaCl}$ ), maintaining the molar ratio of VLPs and Pd NPs at nearly $1: 1$. The solution was mechanically mixed for 3-5 min and keep stored at $4{ }^{\circ} \mathrm{C}$ over night. VP2 VLPs encapsulated Pd NPs, named Pd@VP2, were further purified by dialysis (MWCO: $25 \mathrm{kD}$, SPECTRA/POR, USA) in Tris-HCl buffer (50 mM, pH 8.0) over night.

\subsection{Immobilization of Pd@VP2 on Graphene Oxide Sheets}

Before Pd@VP2 immobilization, GO was prepared through a modified Hummers' method [36, 39]. The prepared GO was then dispersed in water with a concentration of $0.2 \mathrm{mg} / \mathrm{mL}$. After that, $1 \mathrm{~mL}$ of Pd@ VP2 aqueous solution $(0.25 \mathrm{mg} / \mathrm{mL})$ and $1 \mathrm{~mL}$ of $\mathrm{GO}$ aqueous solution was mixed together, keeping stirring for $6 \mathrm{~h}$. Finally, immobilized Pd@ VP2 on GO, labelled as Pd@ VP2/GO, was obtained through centrifugation. The efficiency of Pd@VP2 immobilization (E) was determined via adsorption change of Pd@VP2 in aqueous solution before and after their immobilization on $\mathrm{GO}$ at the wavelength of $280 \mathrm{~nm}\left(\lambda_{280 \mathrm{~nm}}\right)$, as shown in Eq. 1 .

$E=\frac{A_{280}^{0}-A_{280}}{A_{280}^{0}} \times 100 \%$

where $A_{280}^{0}$ and $A_{280}$ are the adsorptions of Pd@ VP2 in aqueous solution before and after their immobilization on GO at $\lambda_{280 \mathrm{~nm}}$, respectively.

After that, Pd@VP2/GO was resuspended in $1 \mathrm{ml}$ of deionized water for the following reaction. 


\subsection{Characterization of Rotavirus VP2 VLPs, Pd@ VP2 and Pd@VP2/G0}

Molecular weight of recombinant rotavirus VP2 protein were tested by sodium dodecyl sulfate polyacrylamide gel electrophoresis (SDS-PAGE). Fast protein liquid chromatography (FPLC) was employed for the purification of Pd@ VP2 nanocages and elution was monitored using a UV-vis spectrometer at the wavelength of $280 \mathrm{~nm}$. Appearance of rotavirus VP2 VLPs, Pd@VP2 and Pd@VP2/GO were negative stained with $2.0 \mathrm{wt} \%$ uranyl acetate and observed by transmission electron microscopy (TEM) (JEOL-2010) at $200 \mathrm{kV}$. Size distribution and zeta potential of Pd colloidal NPs dispersed in the suspension was characterized by a Zetasizer Nano ZES instrument (Malvern Instruments) at $25{ }^{\circ} \mathrm{C}$ using laser with wavelength of $633 \mathrm{~nm}$. Some characteristic functional groups on GO and Pd@VP2/GO were obtained by Fourier transform infrared (FT-IR) spectra, collecting on a Bruker Tensor 27 spectrometer. X-ray photoelectron spectroscopy (XPS) measurements were performed using a Thermo Scientific K-Alpha + X-ray photoelectron spectrometer using $\mathrm{Al} \mathrm{Ka}$ radiation as the excitation source.

\subsection{Catalytic Performance over Pd Catalysts}

The catalytic performance over confined nano catalyst Pd@ VP2 was evaluated in the reduction of 4-nitrophenol (4-NP) to 4-aminophenol (4-AP), shown in Fig. 1. The result was compared to that of non-confined Pd colloidal NPs. The tests were conducted in a 96-well plate at room temperature. Specifically, $30 \mu \mathrm{L}$ of 4-NP aqueous solution $(0.4-4.0 \mathrm{mM})$ and $30 \mu \mathrm{L}$ of Pd@VP2 aqueous solution and $110 \mu \mathrm{L}$ of deionized water were fully mixed in the well of the plate. The reaction began once $30 \mu \mathrm{L}$ of $0.1 \mathrm{~mol} / \mathrm{L} \mathrm{KBH}_{4}$ aqueous solution was added to the mixed solution. The final concentration of $\mathrm{Pd}$ was controlled to $2-8 \mu \mathrm{M}$. Results were monitored on line by microplate reader (Spark $10 \mathrm{M}$, Tecan, Switzerland) from the wavelength of $200 \mathrm{~nm}$ to $450 \mathrm{~nm}$. Reactions were ended when there was no absorption change. Non-confined Pd colloidal NPs were employed as the control. 4-NP and 4-AP have the maximal adsorptions at the wavelengths of 320, and $296 \mathrm{~nm}$, respectively. Noted that, 4-NP solution without $\mathrm{NaBH}_{4}$ has a maximal adsorption at the wavelength of $320 \mathrm{~nm}$, while it is ionized by the addition of excess amount of $\mathrm{NaBH}_{4}$ and its maximal absorption wavelength transfers from 320 to $400 \mathrm{~nm}$ (Fig. S1 in supplementary information, SI). 4-NP reduction will not proceed until the addition of the catalyst. The adsorption at the wavelength of $296 \mathrm{~nm}$ representing 4-AP was not observed in the blank test even after 17 min (Fig. S1), proving this opinion. Hence, the conversion of 4-NP $(X)$ in the reaction was estimated by the decline of adsorption intensity at the wavelength of $400 \mathrm{~nm}$ using Eq. 2.

$X=\frac{A_{N P}^{0}-A_{N P}}{A_{N P}^{0}} \times 100 \%$

where $A_{N P}^{0}$ and $A_{N P}$ are the initial adsorption of 4-NP and the instant one during the reaction at $\lambda_{400 \mathrm{~nm}}$, respectively.

Since 4-NP hydrogenation is generally considered as a pseudo-first-order reaction [30, 40], apparent reaction rate constants $\left(k_{a p p}\right)$ for 4-NP hydrogenation were calculated based on total conversion of 4-HP over Pd catalysts by Eq. 3 .

$\ln \frac{100}{100-X}=k_{\text {app }} \times t$

where $X$ and $t$ represents the total conversion of 4-NP and reaction time, respectively.

The normalized reaction rate constant $\left(k_{n o r}\right)$ was introduced for a quantitative comparison. It was calculated by Eq. 4 .

$k_{\text {nor }}=k_{\text {app }} \times\left(n_{P d}\right)^{-1}$

where $n_{P d}$ represents molar number of Pd.

Initial reaction rates in Turnover frequency (TOF) over Pd@VP2 was further calculated by Eq. 5 and 6.

$$
\begin{aligned}
& \text { initialTOF }=k_{\text {app }} \times n_{N P}^{0} \times\left(n_{\text {surfacePd }}\right)^{-1} \\
& n_{\text {surfacePd }}=n_{P d} \times D_{P d}
\end{aligned}
$$

where $D_{P d}$ is Pd dispersion that could be estimated by spherical model of metal particles; and $n_{N P}^{0}$ is initial molar number

Fig. 1 Reduction of 4-NP to 4-AP

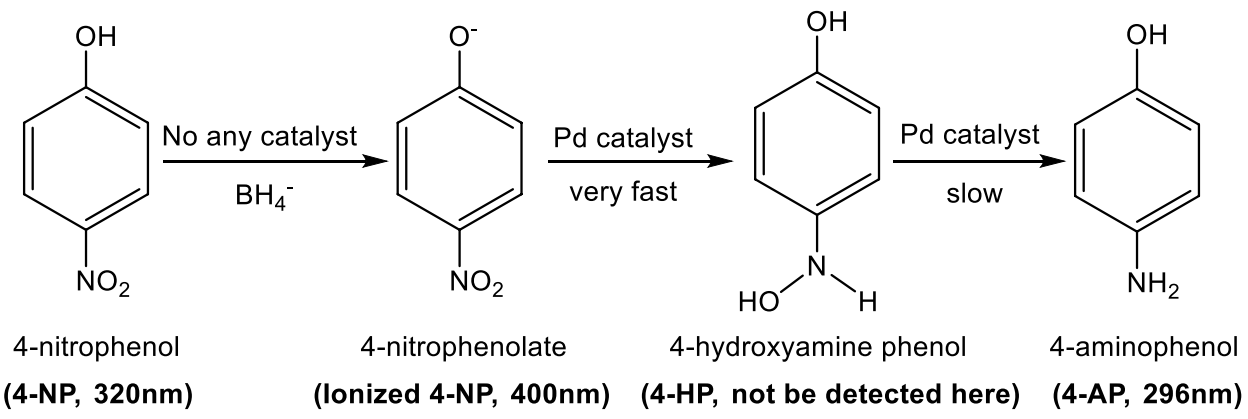


of 4-NP; $n_{\text {surface Pd }}$ is the molar number of surface Pd that could be calculated by Eq. 6 .

Also,immobilized catalyst Pd@VP2/GO was employed for recycling test through four consecutive reactions. At the end of the reaction, Pd@VP2/GO was filtered and rinsed with water for three times at the room temperature. The recycled catalyst was employed for the next run.

\subsection{Analysis in Statistics}

4-NP reduction over Pd catalysts in this work were all carried out with four parallel tests and the results were analyzed in statistics.

\section{Results and Discussion}

\subsection{Synthesis of Pd Nanoparticles}

Metal nanoparticles without any protection prefer agglomeration to large particles if they diffuse freely in the liquid phase due to their high surface energies as well as van der Waals forces among them. Hence, some stabilizers such as polymers or surfactants are often employed surrounding individual nanoparticles to prevent their agglomeration. Herein, PVP (nonionic polymer) and three types of surfactants including CTAB (cationic one), SDS (anionic one) and Tween 80 (nonionic one), were used to control particle sizes of synthesized Pd NPs. As-prepared Pd colloidal NPs were labelled as Pd-PVP, Pd-CTAB, Pd-SDS and Pd-Tween80, respectively.

Properties of above Pd colloidal NPs were summarized in Table 1. Obviously, the order of stabilizers for efficiently controlling the particle size of Pd NPs was Tween $80>$ SDS $>$ PVP $>$ CTAB. Tween 80, the best stabilizer among four ones, for example, had the size distribution in the range of 10-1000 $\mathrm{nm}$ in aqueous medium and its mean size was calculated to be about $37.26 \mathrm{~nm}$ (Fig. S2c, SI). In comparison, Pd-CTAB had the size distribution mainly in the range of 60-1000 nm with the mean size at $233.2 \mathrm{~nm}$. Results closely related to the properties of stabilizers. CTAB, a cationic surfactant, is hard to adsorb on electrophilic Pd NPs surfaces. Hence, it is unfavorable to the stability of Pd

Table 1 Properties of Pd colloids with some stabilizers

\begin{tabular}{lcl}
\hline Sample & $\begin{array}{l}\text { Average size of Pd col- } \\
\text { loid }(\mathrm{nm})\end{array}$ & Zeta potential /mV \\
\hline Pd-CTAB & 233.2 & -0.21 \\
Pd-SDS & 48.66 & -13.5 \\
Pd-Tween80 & 37.36 & -22.3 \\
Pd-PVP & 118.4 & -6.95 \\
\hline
\end{tabular}

NPs (Zeta potential only $-0.21 \mathrm{mV}$ ). However, SDS, an anionic one, is opposite to CTAB. Electrostatic stabilization occurs in this case by adsorbing SDS molecules on the surface of Pd NPs, resulting in coulombic repulsion between the nanoparticles [41]. Apparently, it helps the stability of Pd NPs (Zeta potential-13.5 mV). In addition, Tween 80 and PVP, both nonionic stabilizers, achieve steric stabilization by a protective barrier composed of large molecules. They can chemically bond with some part of Pd surface as well as physically occupy the interspace between Pd NPs, preventing direct contact between them [42]. Actually, Tween 80 stabilized Pd NPs possessed the smallest mean size and the best stability (Zeta potential $-22.3 \mathrm{mV}$ ) as well in this work. Compared to Pd-Tween80, Pd-PVP had the larger mean particle size, very possibly attributing to the polymer with a long chain surrounding individual Pd NPs. Also, negatively charged Pd-Tween 80 favors the encapsulation of it into VLP via self-assembly due to its strong interaction with cationic VP2 capsid proteins. In summary, Tween 80 was selected to stabilize Pd NPs in the following studies.

Pd colloid preparation process was further optimized via varying the molar ratio of $\mathrm{Pd}$ to Tween 80 and results were shown in Fig. 2. In general, with the addition amount of Tween 80 increasing, the mean size of Pd colloidal NPs had a tendency of concave curve. For instance, when the molar ratio of Pd to Tween 80 declined from 1:0.015 to 1:0.1, the corresponding mean size of Pd colloidal NPs greatly decreased from 160 to $16 \mathrm{~nm}$. However, if the molar ratio of Pd to Tween 80 continued descending, its mean size rose up. It was reasonably attributed to the formation of micelles with large sizes that were composed of excessive surfactant molecules in liquid phase. Hence, the optimal molar ratio of the Pd to Tween 80 was 1:0.1 in this work. As-prepared Pd-Tween 80 with the mean size of $16 \mathrm{~nm}$ will be further



Fig. 2 Mean size of Pd colloid as a function of molar ratio of Pd to Tween 80 
Fig. 3 SDS-PAGE analysis of recombinant rotavirus capsid VP2 proteins

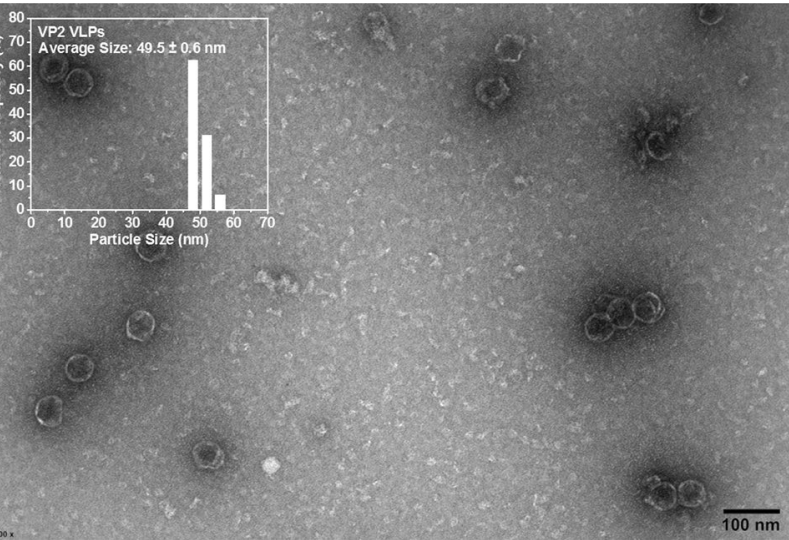

Fig. 4 Self-assembly of recombinant VP2 proteins to VLPs and the size distribution

employed to prepare rotavirus VP2 VLPs confined Pd nano catalyst (Pd@VP2).

\subsection{Encapsulation of Pd NPs into Recombinant Rotavirus Capsid VP2 VLPs}

The expressed and purified recombinant rotavirus capsid VP2 proteins were firstly tested by SDS-PAGE, as shown in Fig. 3. Clearly, VP2 proteins with a high purity (greater than $98 \%$ ) were achieved through purification process. The molecular weight of VP2 was estimated to be about 102 $\mathrm{kD}$, hinting a monomeric form in reducing SDS-PAGE conditions. VP2 proteins can further assemble into spherical VLPs in Tris-HCl buffer (50 mM, pH8.0) [43] with the average size of $49.5 \pm 0.6 \mathrm{~nm}$ (Fig. 4), corresponding to that of the native rotavirus [44]. Figure S3 (SI) indicated a good stability of assembled VP2 VLPs in a wide pH environment
(pH 3.0-9.6). Its average sizes in aqueous solution at $\mathrm{pH}$ 3.0, for example, was measured by dynamic light scattering to be $55.82 \pm 4.52 \mathrm{~nm}$ (Fig. S3a), similar to that at $\mathrm{pH}$ $9.6(53.61 \pm 6.973 \mathrm{~nm}$, Fig. S3c). Results proved the strong self-assembly capability of recombinant VP2 proteins. They favored the encapsulation of metal nanoparticles in them for constructing confined nano catalysts.

Fast protein liquid chromatography (FPLC) was employed for the purification of Pd@VP2 nanocages, as shown in Fig. S4 (SI). Results showed that Pd@VP2 passed through the column with the elution volume at about $10 \mathrm{~mL}$, followed by VP2 VLPs at about $16 \mathrm{~mL}$. TEM image of purified Pd@ VP2 (Fig. 5a) hinted the successful encapsulation of Pd NPs into recombinant rotavirus VP2 VLPs. Most VLPs contained 1-2 Pd NPs with the mean size of $8.15 \pm 0.26 \mathrm{~nm}$. Furthermore, based on adsorption intensity ratio of Pd@VP2 to VP2 VLPs, encapsulation rate was beyond $80 \%$.

\subsection{Immobilization of Pd@VP2 on G0}

As-prepared Pd@VP2 was immobilized on GO sheets by a simple mixing method. Figure $5 \mathrm{~b}$ showed TEM image of their appearance. Clearly, a great number of Pd@VP2 were observed to adsorb on GO (Fig. 5b). The appearance and size of Pd@VP2 maintained unchanged before and after immobilization, hinting the stability of Pd@VP2. The efficiency of Pd@VP2 immobilization was determined via adsorption change of Pd@VP2 in aqueous solution before and after immobilization on GO at the wavelength of $280 \mathrm{~nm}$ $\left(\lambda_{280 \mathrm{~nm}}\right)$, the maximum absorption wavelength of proteins. Based on UV/Vis spectrum in Fig. S5 (SI), Pd@VP2 immobilization efficiency was calculated to be $85 \%$, indicating the high efficiency of this immobilization method.

Some characterizations including FT-IR and XPS were further conducted to investigate adsorption mode of Pd@ VP2 on GO sheets. At first, FT-IR spectra in Fig. 6 identified some functional groups on GO before and after Pd@ VP2 immobilization on it. Specifically, FT-IR spectrum of GO in Fig. 6 showed some typical groups such as $\mathrm{sp}^{2}$ carbon ring $\left(\mathrm{C}-\mathrm{C}, 1610 \mathrm{~cm}^{-1}\right)$, hydroxyl group $(\mathrm{C}-\mathrm{OH}$, $\left.1045 \mathrm{~cm}^{-1}\right)$, epoxy group $\left(\mathrm{C}-\mathrm{O}-\mathrm{C}, 1216 \mathrm{~cm}^{-1}\right)$ and carbonyl group $\left(\mathrm{C}=\mathrm{O}, 1720 \mathrm{~cm}^{-1}\right)$, corresponding to those reported in references $[36,37]$. While after the immobilization of Pd@VP2 on GO, its FT-IR spectrum exhibited strong characteristic peaks of amide II band (C-NH-, $\left.1524 \mathrm{~cm}^{-1}\right)$ and carbonyl group $\left(\mathrm{C}=\mathrm{O}, 1649 \mathrm{~cm}^{-1}\right)$ that were originated from proteins. Noted that, the weak peak of epoxy groups very possibly hinted that they were employed to covalently link to VP2 proteins. Therefore, FT-IR results provided the direct evidence for the successful immobilization of Pd@ VP2 on GO.

Moreover, XPS spectra were analyzed to characterize the surface chemical compositions of Pd@VP2/GO, for 

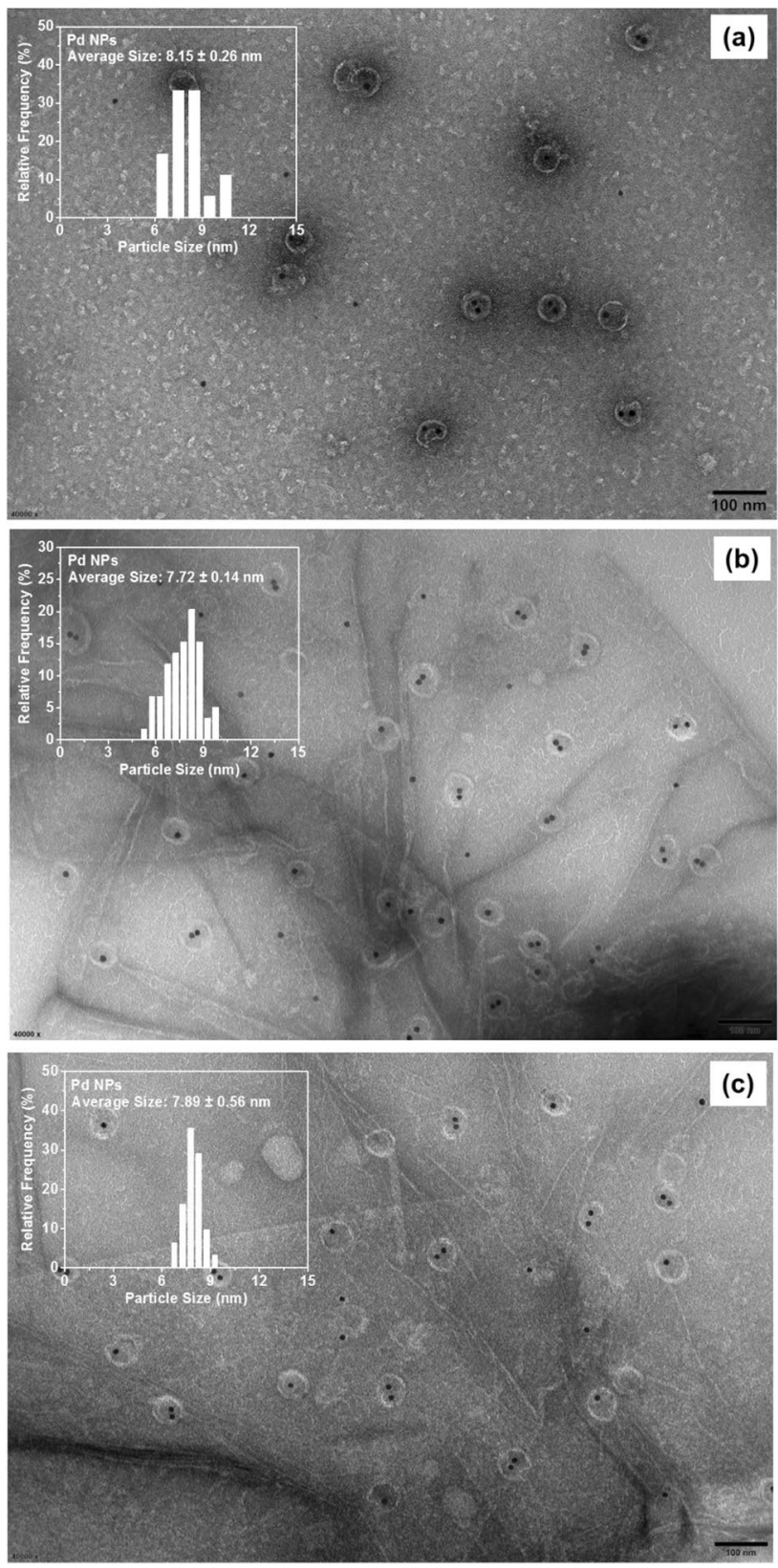

Fig. 5 TEM images of Pd@VP2 (a), Pd@VP2/GO (b) and recycled Pd@VP2/GO (c) with Pd size distributions

testifying its adsorption mode on GO. From Fig. 7a, C1s spectrum of GO was deconvoluted in four characteristic peaks at 284.6, 286.4, 287.3 and $288.5 \mathrm{eV}$, corresponding to $\mathrm{sp}^{2}$ carbon ring, $\mathrm{C}-\mathrm{OH}, \mathrm{C}-\mathrm{O}-\mathrm{C}$ and $\mathrm{C}=\mathrm{O}$ groups, respectively. However, after Pd@VP2 immobilization on GO, its C1s spectrum (Fig. 7b) exhibited a new peak at $285.7 \mathrm{eV}$, representing amine $(\mathrm{C}-\mathrm{N})$ group. It was reasonably originated from covalently linkage of VP2 proteins to GO. Noted that, the molar fraction of $\mathrm{C}-\mathrm{O}-\mathrm{C}$ group in $\mathrm{C} 1 \mathrm{~s}$ distribution was declined from $16.2 \%$ to $7.1 \%$ after Pd@ VP2 immobilization on GO. It proved that $\mathrm{C}-\mathrm{O}-\mathrm{C}$ groups on $\mathrm{GO}$ were



Fig. 6 FT-IR spectra of GO and Pd@ VP2/GO

employed to covalently link to VP2 proteins, in accordance with FT-IR results.

Based on above characterizations, Pd@VP2 were successfully immobilized on GO surface via SN2 reaction. Specifically, the protein cage contains amino groups with lone pair electrons on its surface. As a nucleophilic reagent, protein cage could easily attack electropositive carbon atom of epoxy group on GO surface, leading to the ring opening. Thus, Pd@VP2 were covalently linked to GO surface through $\mathrm{C}-\mathrm{N}$ bond, as shown in Fig. 8.

\subsection{Catalytic Activity of Pd@VP2 on the Reduction of 4-NP}

Active sites of Pd@VP2 were firstly analyzed by Pd3d XPS spectrum (Fig. 7c). Obviously, it showed four typical peaks at $336.2,338.6,341.5$ and $343.7 \mathrm{eV}$, representing $\mathrm{Pd}^{0} 3 \mathrm{~d} 5 / 2$, $\mathrm{Pd}^{2+} 3 \mathrm{~d} 5 / 2, \mathrm{Pd}^{0} 3 \mathrm{~d} 3 / 2$ and $\mathrm{Pd}^{2+} 3 \mathrm{~d} 3 / 2$, respectively. $\mathrm{Pd}^{0}$ species accounted for approximately $86.5 \%$ of the total. The appearance of partial $\mathrm{Pd}^{2+}$ species was possibly attributed to the coordination effect of surfactant on Pd. Oxygen atoms in some functional groups such as ethoxy groups of Tween 80 may provide electrons for anchoring Pd ions, leading to insufficient reduction of $\mathrm{Pd}^{2+}$ on anchoring sites [45-48]. Results further hinted that the addition of Tween 80 favors the dispersion of Pd NPs, preventing their aggregation.

The reduction of 4-NP to 4-AP is a consecutive reaction (Fig. 1). As mentioned in the experimental section, 4-NP is firstly ionized by the addition of excess amount of $\mathrm{BH}_{4}{ }^{-}$. After the further addition of Pd catalyst, 4-NP reduction begins. It is hydrogenated to intermediate 4-HP and then the final one 4-AP. Noted that, the reduction of 4-NP to 4-HP proceeds so fast that 4-HP is hard to detect in this study, as shown in Fig. S6a. Herein, reduction of 4-NP to 4-AP was employed for evaluating the catalytic activity over asprepared catalysts. 
Fig. 7 XPS spectra of GO and Pd@VP2/GO: C1s spectrum of GO (a) and Pd@VP2/GO (b), Pd3d spectrum of Pd@VP2/ $\mathrm{GO}(\mathbf{c})$


<smiles>CC(O)CCC(O)C(C)Nc1ccccc1</smiles>

Fig. 8 Mechanism of Pd@VP2 immobilization on GO via SN2 reaction

Effects of the molar ratio of 4-NP to Pd@VP2 on the reaction were then studied. The concentration of 4-NP was varied from 0.4 to $4 \mathrm{mM}$ and that of Pd in Pd@VP2 solution kept constant at $5 \mu \mathrm{M}$. The reaction proceeded for $24 \mathrm{~min}$. Results indicated that 4-NP conversion rose up with the decrease of 4-NP concentration (Fig. 9). For example, 4-NP conversion was only $(66.12 \pm 1.86) \%$ with 4 -NP concentration at $4 \mathrm{mM}$. However, it arrived at $(98.14 \pm 1.72) \%$ when 4-NP concentration declined to $1.6 \mathrm{mM}$ at the same time (Experimental data in Table S1, SI). Clearly, excessive substrates in the catalytic system led to the complete reaction for a longer time. Nevertheless, when 4-NP concentration decreased to lower than $1.6 \mathrm{mM}, 4-\mathrm{NP}$ conversion fell very slowly in this study due to the enough Pd sites for the reaction in this case. Therefore, 4-NP with the concentration at $1.6 \mathrm{mM}$ was chose for the following reactions.

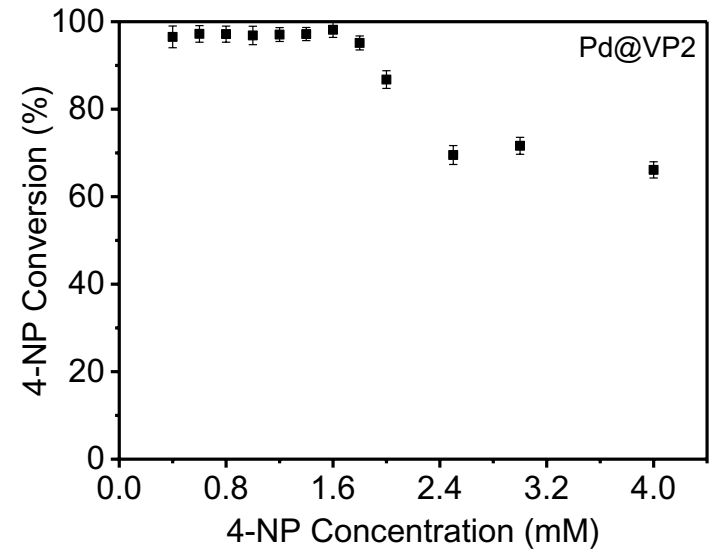

Fig. 9 4-NP conversion as a function of 4-NP concentration over Pd@VP2. (Statistics: mean value of four parallel tests \pm standard error)

Moreover, the dependence of reduction rate constant with respect to the catalyst concentration was investigated here. The final concentration of Pd in Pd@VP2 was controlled to $2-8 \mu \mathrm{M}$ and that of $4-\mathrm{NP}$ kept constant at $1.6 \mathrm{mM}$. The reaction proceeded for $24 \mathrm{~min}$. The result was shown in Fig. 10. Obviously, $k_{a p p}$ climbed up with the concentration of the catalyst increasing. It was only $(1.04 \pm 0.05) \times 10^{-3} \mathrm{~s}^{-1}$ with final Pd concentration at $2 \mu \mathrm{M}$, while $k_{a p p}$ was up to 




Fig. 10 Apparent rate constant as a function of $\mathrm{Pd}$ concentration in Pd@VP2. (Statistics: mean value of four parallel tests \pm standard error)

$(3.94 \pm 0.11) \times 10^{-3} \mathrm{~s}^{-1}$ when the final Pd concentration increased to $6 \mu \mathrm{M}$. Noted that, $k_{\text {app }}$ values hardly changed with the final Pd concentration increasing from 5 to $8 \mu \mathrm{M}$ in this study. It hinted the highest efficiency of Pd@VP2 with the final Pd concentration at 5-6 $\mu \mathrm{M}$ in this catalytic system.

Based on above results, 4-NP reduction on Pd catalyst proceeds very possibly in terms of Langmuir-Hinshelwood kinetics, in agreement with some reported literatures [49-51, 56]. In this mechanism, both $\mathrm{BH}_{4}{ }^{-}$and ionized 4-NP in the catalytic system are firstly adsorbed on the surface of $\mathrm{Pd}$ NPs. $\mathrm{BH}_{4}^{-}$, as a strong reducer, is then decomposed on $\mathrm{Pd}$ surface to form Pd-hydride. It provides activated hydrogen and electrons to nitro groups of 4-NP adsorbed on Pd surface, resulting in the reduction of nitro groups to amino ones. After that, as-synthesized 4-AP was desorbed from the surface of Pd NPs to the bulk.

Finally, we compared the catalytic performance of Pd@VP2 in 4-NP reduction to those of non-confined PdTween 80 and some previously reported Pd-based catalysts (Table 2) [52-56], for investigating its potential prospect in catalysis. Figure 11 hinted an enhanced activity of Pd NPs after the encapsulation of them into VLPs. 4-NP conversion over Pd-Tween80, for instance, was $(91.56 \pm 2.23) \%$ after $1440 \mathrm{~s}$ reaction (Fig. 11a). In comparison, that over Pd@VP2 reached $(98.14 \pm 1.62) \%$ at the same time. Its apparent reaction rate constants $\left(k_{\text {app }}\right)$ was calculated to be $(3.74 \pm 0.10) \times 10^{-3} \mathrm{~s}^{-1}, 1.7$ times higher than that over Pd-Tween80 (Fig. 11b). Additionally, the normalized rate constant $\left(k_{n o r}\right)$ and turnover frequency (TOF) for Pd@ VP2 in the reaction were further estimated to be about $224 \mathrm{~min}^{-1} \cdot \mu \mathrm{mol}^{-1}$ and $33 \mathrm{~min}^{-1}$, respectively. Clearly, Pd@VP2 exhibited comparatively high activity in contrast to most of Pd-based catalysts listed in Table 2. It was logically attributed to the confinement effects including the high dispersion of Pd NPs and high effective concentration of substrates in confined space.

\subsection{Recycling of Pd@VP2/GO}

After immobilization of Pd@VP2 on GO, its activity was examined by recycling it for four runs. Results exhibited a slight decline on catalytic activity (Fig. 12). 4-NP conversion after $1440 \mathrm{~s}$ reaction, for instance, decreased from about 86 to $79 \%$ in four runs. It was mainly due to the partial mass loss during the recycling process (about 3\% for each run). $k_{a p p}$ of the recycled Pd@VP2/GO in the fourth run was further calculated to be $(2.01 \pm 0.11) \times 10^{-3} \mathrm{~s}^{-1}$ (Fig. S7). Considering the immobilization efficiency and the mass loss of the catalyst after recycling, the molar number of Pd NPs in recycled Pd@VP2/GO was only about 70\% of that before Pd@VP2 immobilization. Hence, $k_{\text {nor }}$ of recycled Pd@VP2/GO was calculated to be $(2.87 \pm 0.16) \mathrm{s}^{-1} \cdot \mu \mathrm{mol}^{-1}$, maintaining a high activity. However, it exhibited a slightly declined activity in contrast to Pd@VP2 before immobilization $\left((3.74 \pm 0.10) \mathrm{s}^{-1} \cdot \mu \mathrm{mol}^{-1}\right)$. We speculated that it was possibly related to the small part passivation of the catalyst after several runs of catalysis.

Moreover, the stability of Pd@ VP2 after recycling was tested, for investigating the reusability of Pd@VP2/GO. TEM image of Pd@VP2 after four runs (Fig. 5c) exhibited the similar appearance to that before reaction (Fig. 5b). It proved the strong anchoring effects of GO on Pd@VP2, in accordance with XPS analysis. The average size of Pd NPs in Pd@VP2 was measured to be $7.89 \pm 0.56$ nm (inner

Table 2 Comparison of catalytic performance over several Pd-based catalysts for 4-NP reduction

\begin{tabular}{|c|c|c|c|c|c|}
\hline Catalyst & $\begin{array}{l}\text { Mole ratio of } \\
4-N P \text { to Pd }\end{array}$ & $k_{\text {app }}\left(\mathrm{min}^{-1}\right)$ & $k_{\text {nor }}\left(\mathrm{min}^{-1} \mu \mathrm{mol}^{-1}\right)$ & $\operatorname{TOF}\left(\mathrm{min}^{-1}\right)$ & References \\
\hline Pd embedded in polypyrrole nanocapsules (Pd/PPy) & 4 & 0.53 & 9 & 5 & {$[52]$} \\
\hline $\mathrm{PdP}$ on carbon nanospheres (PdP/CNSs) & 77 & 0.11 & 28 & 8 & {$[53]$} \\
\hline Yolk-shell Pd-N-doped carbon (YS-Pd-C/N) & 81 & 0.42 & 114 & 42 & {$[54]$} \\
\hline Pd on iron-rich coal fly ash/silica (Pd/IRFA@ $\left.\mathrm{SiO}_{2}\right)$ & 32 & 14.97 & 1497 & - & {$[55]$} \\
\hline Pd on porous polyurea microspheres (Pd@ PPM2) & 6 & 0.48 & 3 & 1 & {$[56]$} \\
\hline Pd@VP2 & 48 & 0.22 & 224 & 33 & This work \\
\hline
\end{tabular}





Fig. 11 Catalytic performance of Pd-Tween80 and Pd@VP2: a 4-NP conversion versus reaction time; $\mathbf{b}$ estimation of reaction rate constants $(k)$. (Statistics: mean value of four parallel tests \pm standard error)



Fig. 12 Recycling of Pd@VP2/GO for four runs. (Statistics: mean value of four parallel tests \pm standard error)

graph of Fig. 5c), keeping unchanged in comparison to that before reaction $(7.72 \pm 0.14 \mathrm{~nm}$, inner graph of Fig. $5 \mathrm{~b})$. It hinted the high stability of Ru NPs with the protection of protein nanocages. Zeta potential of Pd@VP2/GO after four runs was measured to be $-36.1 \pm 9.8 \mathrm{mV}$ (Fig. S8b), slightly less than that before reaction $(-38.7 \pm 9.77 \mathrm{mV}$, Fig. S8a), possibly owing to the adsorption of a small amount of salt cations on GO surface. Results exhibited the strong electrostatic repulsions among Pd@VP2/GO particles. It favored the dispersion of them in aqueous solution, maintaining the high catalytic activity even after several runs. Additionally, Pd@VP2/GO particles after four runs possessed the mean size of $712.4 \mathrm{~nm}$ (Fig. S9b), similar to that before reaction $(693.3 \pm 33.54 \mathrm{~nm}$, Fig. S9a). It proved the stability of asprepared Pd@VP2/GO, indicating its reusable potential in catalysis.

\section{Conclusions}

In summary, we synthesized a confined nanoreactor by encapsulating Tween 80 stabilized Pd nanoparticles into rotavirus VP2 VLPs (i.e. Pd@VP2). In comparison to nonconfined Pd-Tween80, Pd@VP2 exhibited an enhanced activity in the reduction of 4-NP to 4-AP thanks to the confinement effects including the high dispersion of Pd NPs and high effective concentration of substrates in confined space. Furthermore, Pd@VP2 was immobilized on GO sheets (i.e. Pd@VP2/GO) via a simple mixing method. It showed a slight decline on catalytic activity across four runs, hinting the stable and reusable capability of Pd@VP2/GO. This work potentially created a facile and environment-friendly route to construct an immobilized catalytic nanoreactor based on viral protein cages with the cheerful prospects in sustainable and green chemistry.

Acknowledgements This work was supported by the National Natural Science Foundation of China (No. 21676029). The financial support from the Priority Academic Program Development of Jiangsu Higher Education Institutions (PAPD) is also acknowledged.

\section{References}

1. Santiso EE, Kostov MK, George AM, Nardelli MB, Gubbins KE (2007) Appl Surf Sci 253:5570-5579

2. Stein A, Rudisill Stephen G, Petkovich ND (2014) Chem Mater 26:259-276

3. Chrissopoulou K, Anastasiadis SH (2015) Soft Matter 11:3746-3766

4. Leenders SHAM, Gramage-Doria R, de Bruin B, Reek JNH (2015) Chem Soc Rev 44:433-448

5. Zhang Z, Xu B, Wang X (2014) Chem Soc Rev 43:7870-7886

6. Cheng L, Jiang X, Wang J, Chen C, Liu R (2013) Nanoscale 5:3547-3569

7. Li G, Tang Z (2014) Nanoscale 6:3995-4011

8. Yabu H, Higuchi T, Jinnai H (2014) Soft Matter 10:2919-2931

9. Dai W, Zhang S, Yu Z, Yan T, Wu G, Guan N, Li L (2017) ACS Catal 7:3703-3706

10. Göltl F, Michel C, Andrikopoulos PC, Love AM, Hafner J, Hermans I, Sautet P (2016) ACS Catal 6:8404-8409 
11. Janda A, Vlaisavljevich B, Lin L, Smit B, Bell AT (2016) J Am Chem Soc 138:4739-4756

12. Xiao J, Pan X, Zhang F, Li H, Bao X (2017) Chem Sci 8:278-283

13. Deng D, Chen X, Yu L, Wu X, Liu Q, Liu Y, Yang H, Tian H, Hu Y, Du P, Si R, Wang J, Cui X, Li H, Xiao J, Xu T, Deng J, Yang F, Duchesne PN, Zhang P, Zhou J, Sun L, Li J, Pan X, Bao X (2015) Sci Adv 1:e1500462

14. Miners SA, Ranc GA, Khlobystov AN (2013) Chem Commun 49:5586-5588

15. Hernández-Rojas J, Calvo F, Bretón J, Llorente JMG (2012) J Phys Chem C 116:17019-17028

16. Ha B, Jung Y (2015) Soft Matter 11:2333-2352

17. Smyda MR, Harvey SC (2012) J Phys Chem B 116:10928-10934

18. Rathfon JM, Cohn RW, Crosby AJ, Rothstein JP, Tew GN (2011) Macromolecules 44:5436-5442

19. Brunet G, Safin DA, Robeyns K, Facey GA, Korobkov I, Filinchuk Y, Murugesu M (2017) Chem Commun 53:5645-5648

20. Wang Y, Cui H, Wei Z, Wang H, Zhang L, Su C (2017) Chem Sci 8:775-780

21. Jiang Y, Zhang X, Dai X, Zhang W, Sheng Q, Zhuo H, Xiao Y, Wang H (2017) Nano Res 10:876-889

22. Heinzelmann G, Figueiredo W (2016) J Chem Phys 145:164902

23. Durantini AM, Falcone RD, Silber JJ, Correa NM (2016) Chem Phys Chem 17:1678-1685

24. Pradham S, Bartley JK, Bethell D, Golunski SE, Hutchings GJ (2012) Catal Lett 142:302-307

25. de la Escosura A, Nolte RJM, Cornelissen JJLM (2009) J Mater Chem 19:2274-2278

26. Luo Q, Hou C, Bai Y, Wang R, Liu J (2016) Chem Rev 116:13571-13632

27. Yang L, Liu A, Cao S, Putri RM, Jonkheijm P, Cornelissen JJLM (2016) Chem Eur J 22:1-14

28. Douglas T, Young M (2006) Science 312:873-875

29. Fischlechner M, Donath E (2007) Angew Chem Int Ed 46:3184-3193

30. Liu A (2016) Traulsen CH-H, Cornelissen JJLM. ACS Catal 6:3084-3091

31. Liu A, Yang L (2017) Traulsen CH-H, ornelissen JJLM. Chem Commun 53:7632-7634

32. Steen Redeker E, Ta DT, Cortens D, Billen B, Guedens W, Adriaensens P (2013) Chem 24:1761-1777

33. Han Y-L, Gao P-J, Cheng C-L, Wu P-Y, Chang J-S (2019) Biochem Eng J 152:107388-107393

34. Wang Y, Rong Z, Wang Y, Qu J (2016) J Catal 333:8-16

35. Sun Z, Rong Z, Wang Y, Xia Y, Du W, Wang Y (2014) RSC Adv $4: 1874-1878$
36. Zhu J, Ding X, Li D, Dou M, Lu M, Li Y, Luo F (2019) ACS Appl Mater Interfaces 11:16443-16451

37. Zhu J, Gu Y, Wu J, Zhu X, Xue B, Li Y (2017) Catal Lett 147:335-344

38. Li T, Lin H, Zhang Y, Li M, Wang D, Che Y, Zhu Y, Li S, Zhang J, Ge S, Zhao Q, Xia N (2014) Vaccine 32:1921-1931

39. Liu F, Sun J, Zhu L, Meng X, Qi C, Xiao FS (2012) J Mater Chem 22:5495-5502

40. Baran NY, Baran T, Nasrollahzadeh M, Varma RS (2019) J Organomet Chem 900:120916-120925

41. Aiken JD III, Finke RG (1999) J Mol Catal A-Chem 145:1-44

42. Ott LS, Finke RG (2007) Coord Chem Rev 251:1075-1100

43. Ge S, Li T, Guo Q, Xu F, Zhang J, Xia N. Method for in-vitro preparation of double-layered virus-like particles of rotavirus. U.S. Patent 986,293,3B2, January 09, 2018.

44. Pesavento JB, Crawford SE, Estes MK, Prasad BV (2006) Curr Top Microbiol 309:189-219

45. Liz-Marzán LM, Lado-Touriño I (1996) Langmuir 12:3585-3589

46. Fan M, Long Y, Zhu Y, Hu X, Dong Z (2018) Appl Catal A: Gen 568:130-138

47. Arrigo R, Schuster ME, Xie Z, Yi Y, Wowsnick G, Sun LL, Hermann KE, Friedrich M, Kast P, Hävecker M, Knop-Gericke A, Schlögl R (2015) ACS Catal 5:2740-2753

48. Cui X, Long Y, Zhou X, Yu G, Yang J, Yuan M, Ma J, Dong Z (2018) Green Chem 20:1121-1130

49. Nemanashi M, Meijboom R (2016) Catal Commun 83:53-57

50. Zhang X, Fan L, Cui Y, Cui T, Chen S, Ma G, Hou W, Wang L (2020) NANO 15:2050002

51. Nemanashi M, Meijboom R (2013) J Colloid Interf Sci 389:260-267

52. Xue Y, Lu X, Bian X, Lei J, Wang C (2012) J Colloid Interf Sci 379:89-93

53. Zhao Z, Ma X, Wang X, Ma Y, Liu C, Hang H, Zhang Y, Du Y, Ye W (2018) Appl Surf Sci 457:1009-1017

54. Long Y, Liu Y, Zhao Z, Luo S, Wu W, Wu L, Wen H, Wang R, Ma J (2017) J Colloid Interf Sci 496:465-473

55. Park J, Bae S (2019) J Hazard Mater 371:72-82

56. Bashir MS, Jiang X, Kong XZ (2020) Eur Polym J 129:109652

Publisher's Note Springer Nature remains neutral with regard to jurisdictional claims in published maps and institutional affiliations. 